\title{
An Approach to Cellular Tropism of SARS-Cov-2 Through Protein-Protein Interaction and Enrichment Analysis
}

\section{Daniel Ortega-Bernal}

Universidad Autónoma Metropolitana

\section{Selene Zarate}

Universidad Autónoma de la Ciudad de México

Maria de los Ángeles Cárdenas

Universidad Autónoma Metropolitana

Rafael Bojalil ( $\nabla$ rbojalil@correo.xoc.uam.mx)

Universidad Autónoma Metropolitana

\section{Research Article}

Keywords: COVID-19, SARS-CoV-2, pulmonary disease, cellular-level tropism, proteomics, pneumocytes

Posted Date: August 27th, 2021

DOI: https://doi.org/10.21203/rs.3.rs-831421/v1

License: @ (i) This work is licensed under a Creative Commons Attribution 4.0 International License. Read Full License 


\section{Abstract}

COVID-19, caused by SARS-CoV-2, is a primarily pulmonary disease that can affect several organs, directly or indirectly. To date, there are many questions about the different pathological mechanisms. Here, we generate an approach to identify the cellular-level tropism of SARS-CoV-2 using human proteomics, virus-host interactions, and enrichment analysis. Through a network-based approach, the molecular context was visualized and analyzed. This procedure was also performed for SARS-CoV-1. We obtained proteomes and interactomes from 145 different cells corresponding to 57 different tissues. Not all cells had proteins such as ACE2 or TMPRSS2 (among others), so they were discarded. Of the remaining cells, a gradient of susceptibility to infection was observed. In addition, proteins associated with the coagulation cascade that can be directly or indirectly sequestered by viral proteins were identified. We have identified 55 potential cells that can be "cracked" with different susceptibilities. One of the main results being pneumocytes, as well as heart, kidney, liver, or small intestine. We also report how the coagulation cascade can be affected by SASR-CoV-2 infection. These results help us to explain the molecular context and provide elements for possible treatments in the current situation.

\section{Introduction}

Understanding the molecular interactions of SARS-CoV-2 with its host (cell) is necessary to identify the various impacts of the infection at the organismal level. Sequestration of a host protein by a SARS-CoV-2 protein, either to inhibit or to promote its function, can modify a given molecular process. Taking the numerous protein-protein interactions (PPIs) reported by Gordon DE et al. ${ }^{1}$, Li et al. ${ }^{2}$, and Stukalov et al. ${ }^{3}$, as well as other groups, we have analyzed how 29 SARS-CoV-2 proteins interact with human proteins under their cellular context. They represent an overview of the PPI networks and molecular processes under the context of the cell line from which those authors obtained their results (HEK293T, embryonic kidney and A549 human lung (carcinoma)), as well as some extrapolations. Also, using data from these investigators, simulated data, associations with interactomes of other viruses or experimental data, several groups have generated general molecular contexts between viral proteins and human proteins. Sarbecovirus-human protein-protein interaction network ${ }^{4}$; network-based model for defining the molecular aspects of pathogenic phenotypes in $\mathrm{HCoV}$ infections ${ }^{5}$; overview of coronavirus-host interactions ${ }^{6}$; cellular infection profile ${ }^{7}$; uncover structural and functional modules ${ }^{8}$ or an organotropism beyond the respiratory tract ${ }^{9}$ for example.

Just because a viral particle can enter a cell does not mean that there is an enabling environment for its replication; a coordinated cellular sequestration is necessary. With this research, we have identified a set of cells as potential targets of SARS-CoV-2. First, by identifying those with entry mechanisms, already reported at the tissue level ${ }^{10-18}$. Subsequently, by identifying proteomes and interactomes within each cell associated with viral proteins. The possible alterations of protein-protein interactions (PPI) by the infection within each cell were identified, resulting in a group of cells with a certain degree of 
susceptibility to be "cracked" by SARS-CoV-2. At the same time, we investigated and compared the latter with SARS-CoV-1.

\section{Results}

\section{PPI}

To analyze the interactions between SARS-CoV-2 and cell proteins data from the proteinAtlas were collected as described in the Methods section. A total interactome of 499,620 interactions (edges) and 20,091 proteins (nodes) was generated and then reduced to interactions reported two or more times, resulting in 77,351 PPIs and 13,035 proteins.

\section{Input tropism}

Of the 62 tissues reported in the RNA consensus tissue gene data, 57 had at least three expressed genes of the seven inputs (a combination of protein S-binding proteins (ACE2, NRP1 or BSG) and proteases (TMPRSS2, CTSL, CTSB or FURIN)). Of the 57 tissues ( 145 cell types) reported in the normal tissue data, 45 had at least 3 of the seven proteins we looked for. Both data sets shared 39 tissues, containing 69 different cell types (see Figs. 1 and 2b). Six cell types such as bronchus, nasopharynx or oral mucosa did not have a transcriptome report at the tissue level; 23 tissues had no information for cell types, such as the olfactory region. With SARS-CoV-1, the results were similar, but considering four proteins (ACE2, BSG, TMPRSS2 and CTSL).

Of the 39 tissues identified in the analysis for SARS-CoV-2, 10 (16 cell types) did not report the presence of ACE2 and TMPRSS2 protein, nor mRNA: adrenal gland (glandular cells), bone marrow (hematopoietic cells), cerebellum (granular layer cells, molecular layer cells and Purkinje cells), cerebral cortex (endothelial cells, glial cells, neuronal cells and neuropil), endometrium (glandular cells 1 and 2), germinal and non-germinal center cells of lymph nodes, parathyroid gland (glandular cells), skeletal muscle (myocytes), smooth muscle (smooth muscle cells) and spleen (red pulp cells), see Fig. 2a. Figure. 2a shows that bone marrow did not express ACE2 or TMPRSS2, and several tissues had below threshold values $(\mathrm{NX}<1)$ of ACE2, such as lung (0.8). At this point, no cell type was removed. In contrast, for SARSCoV-1, we removed 14 cell types with neither ACE2 nor TMPRSS2 (nor in MRNA); BSG and CTSL only had expression data from mRNA (see Fig. 2c). These results show that ACE2 and TMPRSS2 are the least present in the 69 cell types of the seven input proteins.

\section{PPI tropism}

To investigate differences in the PPI networks that could occur in infected cells, we compared the size of the interactomes inferred in each cell type. The cells showing the highest number of interactions with SARS-CoV-2 proteins were in the kidney (tubule cells and glomerulus cells), small intestine (glandular cells) and lung (pneumocytes), with a maximum of 970 proteins out of the 1345 reported. The least connected cells are bone marrow (hematopoietic cells), colon (peripheral nerve/ganglion), placenta 
(decidual cells) and ovary (follicular cells), see Supplementary Table S1. In SARS-CoV-1, the results for the cells with the least interaction are the same as in SARS-CoV-2, and for the cells with the most interaction are lung (pneumocytes) and glandular cells (thyroid gland, duodenum, salivary gland, small intestine, gall bladder, colon, and pancreas), with 114 proteins (out of 151) interacting with the 26 viral proteins (see Supplementary Table S2).

Initially it was thought to be an infection associated with an expression profile. When clustering the cell types by expression value, a group of cells was identified with a higher presence of several proteins, see Supplementary Fig. S1. Comparing the unsupervised hierarchical clustering, in Supplementary Fig. S2, mainly these cell types were glandular. A similar pattern was observed for SARS-CoV-1 (Supplementary Fig. S1c). In addition to glandular cells, this clustering shows group cells according to tissue type (skin, brain).

To characterize the dynamics of each cell according to their IPPs, their networks were represented. Obtaining the Average Degree (ranging from 4.561 to 7.391) to identify the connectivity of each network, we determined that the most connected cellular networks are pneumocytes, glandular cells (endometrium 1), squamous epithelial cells (esophagus), non-germinal center cells (lymph node and tonsil tissues) and cells in red pulp (spleen), see Supplementary Table S1. For example, to visualize emblematic cells, in Fig. 3 the lung (pneumocytes and macrophages) as the most affected tissue, and small intestine (glandular cells) as the tissue with the highest expression of ACE2, networks are visualized at the V-H PPI level. This considering the expression in each cell and the total connectivity of each protein. It is observed that the most linked proteins of SARS-CoV-2 are M, nsp7b and orf3a, while the most connected to human are LARP7 and GOLGA2. If we focus on V-H, direct PPIs (without $\mathrm{H}-\mathrm{H}$ edges), the most connected are ATP1A1, ATP2A2, HACD3, XP01 and ATP5F1B. As identified in Supplementary Fig. S1 and Fig. S2, glandular cells (small intestine) show increased expression of several proteins. In Fig. 3G, $3 \mathrm{H}$ and $3 \mathrm{l}$, the networks of the same tissues in SARS-CoV1 are shown, showing UBC as the protein with the most connections, identifying a similar expression pattern as with SARS-CoV-2.

For each proteome of each cell type, a gene set enrichment analysis (GSEA) was generated. Unsupervised clustering (binary distance and the simple linkage method) allowed the identification of a "gradient" of cells, where molecular processes are altered from lower to higher degree. Figure 4 shows the KEGG signaling pathways in SARS-CoV-2 and SARS-CoV-1. In both heatmaps, an effect of proteome size was identified (Supplementary Table S1 y S2), thus 14 cell types were removed, reducing to 55 the initial 69 cell types. For SARS-CoV-1, lung (macrophages) and tonsil (squamous epithelial cells) were also identified as not significantly altering the molecular environment and were therefore also removed for subsequent analyses. Hence, for SARS-CoV-2, 55 cell types remained and for SARS-CoV-1, 45. Supplementary Figure S3 shows the behavior of the remaining SARS-CoV-2 cells in terms of molecular functions and biological processes. With these results, we identified that salivary gland (glandular cells), esophagus (squamous epithelial cells), testis (Leydig cells), lung (pneumocytes) and small intestine (glandular cells) behave as a group (identifiable in molecular functions, dark blue branch, where they 
share a group of unaltered functions). Supplementary Figure S4 shows the effect of this reduction on SARS-CoV-1.

Supplementary Figure $\mathrm{S} 5$ shows the 50 most discriminating results, and the profiles of the "gradients" shown in the previous results can be identified. For SARS-CoV-2, one signaling pathway that appears relevant is "Ribosome biogenesis in eukaryotes" (Supplementary Fig. S5b). Given its relevance, subnetworks of ribosomes and their biogenesis were generated. The ribosome network shows a profile like that observed in Fig. 4 and Supplementary Fig. S1: high protein expression value in the small intestine (glandular cells); moderate-high in the lung (macrophages); moderate in the kidney (tubule cells) and moderate-low in the lung (pneumocytes). Looking at Ribosome biogenesis networks in eukaryotes, we identified that the behavior of pneumocytes is more like that of the small intestine (glandular cells), especially in the Expression value, as is the case of XP01, which is highly connected by SARS-CoV2 proteins, see Fig. 5.

Like ribosome biogenesis in eukaryotes, the coagulation cascade is reported in several results, Fig. 4a. Both signaling pathways are shown in Fig. 6 to visualize the degree of involvement at the pneumocyte and systemic level. In ribosome biogenesis in eukaryotes, FGA, FGB, PROS1, PLAU, PLAT, PROCR, and CPB2 interact directly with virus proteins; with 20 proteins interacting indirectly. In the coagulation cascade, XP01, CSNK2B, XRN2, DKC1, NOP56, NVL, RBM28, NAT10, TBL3, and MPHOSPH10 are proteins that interact directly with virus proteins.

Obtaining the centroids of 55 SARS-CoV-2 cell types ( 45 for SARS-CoV- 1 ), 8 clusters were identified, separated into 4 quadrants. According to the distribution of Expression value and PPI, the importance of connectivity associated with the protein level was identified. Quadrants 1 and 4 (high PPI) of Fig. 7 are the most important, where most of the cells of the tissues most reported in the literature are located, such as pneumocytes, kidney (cells in tubules and cells in glomeruli), testis (cells in seminiferous ducts), cardiac muscle (myocytes), and liver, among others.

\section{Discussion}

In this work, we analyzed human PPI and proteome, GSEA information. We report virus-host protein interactions to identify cells and tissues with the putative necessary environment to sustain infection. This analysis identified a series of tissues and cell types that could potentially be a target for viral infection. Several studies have been carried out to determine the breadth of SARS-CoV-2 cell tropism outside the respiratory tract (e.g., lungs and trachea). Still, many have evaluated the permissiveness of various cell lines, which is hard to interpret. Autopsy analyses have shown the presence of SARS-CoV-2 antigens, by immunodetection, in the trachea, small intestine, the kidneys, pancreas, and vascular tissues of the brain and heart ${ }^{19,20}$. Our work identified as possible targets for infection, in addition to some cells of the respiratory tract, cells from the small intestine and pancreas. Other studies have pointed to the potential infection of these tissues. Interestingly, Stillfried et al. using pathological specimens, have identified SARS-CoV-2 by RT-PCR in different tissues, including the small intestine ${ }^{21}$. 
Initially, it was difficult to identify a set of cells that appear to be susceptible to SARS-CoV-2 infection, although this was not the case for SARS-CoV-1. The amount of ACE2 in the tissues ${ }^{15,16,18}$ has not shown a correlation with infection or correlation with TMPRSS2 concerning all organs reported. Therefore, we decided to take the group of 7 proteins already pointed out, such as NRP1 ${ }^{10}$, that could explain the tropism to different organs. This strategy also did not generate a good relation between these, and the organs affected by SARS-CoV-2. The next step was the intracellular molecular context.

An infection-associated expression profile was suspected, but the overexpression observed is linked to glandular cells, overexpression already identified ${ }^{22,23}$. However, it is plausible to think that the higher the concentration of cell proteins the greater the possibility of interacting with viral proteins, which does not seem to be a trivial relationship since competition for protein interaction could play an important role. This possibility should be explored experimentally but to date there are no results that demonstrate the susceptibility to infection of these cells. On the other hand, the data shown in our results indicate some gradient of molecular involvement, and therefore susceptibility to invasion. Considering these results together with the availability of entry proteins, the concordance of connectivity, and the previously reported autopsy results, supported the hypothesis of a gradient of susceptibility to infection.

Several of the cells identified in this study were expected given the impact seen clinically and from various investigations, e.g., part of the stomatological apparatus ${ }^{24}$, digestive tract, or pneumocytes. Others like cerebral cortex, skin, appendix (glandular cells), tonsil and others, were not expected and should be considered for further study. This amplitude in tropism could be related to the possibility of generating diverse autoantigens, which would contribute to a severe ${ }^{25}$ and long ${ }^{26,27}$ COVID state and the diversity of symptoms this represents. Where in some cases, by having an inefficient capacity to infect certain cells (those that least alter the molecular environment or that the connectivity in the PPI is low), no symptoms occur due to the few infected cells; but under the context of overactivation of the immune system and/or cell lysis several autoantigens can be generated and therefore, the illness can progress and go from a moderate disease to a long COVID-19 28 .

A relevant finding to consider in both the acute disease and the long COVID syndrome is the possible deregulation of ribosome biogenesis due to its critical role in cell function and in diseases such as cancer ${ }^{29}$. In addition to intracellular molecular alterations, such as ribosome biogenesis, already reported in SARS-CoV-1 by PPI ${ }^{30}$, we show a set of coagulation cascade proteins interacting directly and indirectly with SARS-CoV-2 proteins, which could promote their dysregulation. This, together with pro-thrombotic autoantibodies ${ }^{25}$ and other potential causes ${ }^{31}$, may account for explaining a coagulation-promoting environment affecting different organs ${ }^{32}$.

A relevant result in SARS-CoV1 was that lung macrophages could be identified as cells in which the molecular environment may not be significantly altered, and therefore, cell sequestration would be inefficient. This agrees with previous research ${ }^{33,34}$, where it was identified that the viral particle could enter the cell but not replicate, which may also be occurring with several SARS-CoV-2 infected cells. The 
tonsil (squamous epithelial cells) was also eliminated by the same criteria, but no reports in the literature for this case.

In conclusion, a set of 55 cell types whose molecular environment can be altered by SARS-CoV-2 has been identified with a connectivity gradient that allows identification of which of them are the most susceptible to "cracking". As expected, pneumocytes are one of them. We also identified several proteins of the coagulation cascade directly and indirectly affected by viral proteins, pinpointing the effects at this level. Other possible targets of infection identified were cells from the small intestine, colon, stomach, and pancreas; heart, kidney, salivary glands, appendix, and tonsils; testicles, prostate, and endometrium among others, not necessarily expected. We have also identified a profile of molecular alterations in the form of a "gradient" of infection. This model is still perfectible, but it represents an approach to determine the cell tropism of SARS-CoV-2 and other viruses, as was the case with SARS-CoV-1. We expect that this model will contribute to the fight against current and future pandemics by identifying proteins essential for infection, both those of the virus and those of the host.

\section{Limitations}

Several results that have been reported to have clinical significance were not considered. The lack of information on certain cell types and of availability of proteome or transcriptome information meant that not all expected results were obtained, as well as some other hypothetical ones. Cells with a small proteome, such as ovarian (follicular cells) or placental (decidual cells) were also discarded. Whether this is due to a truly small proteome or a technical inability to identify various proteins in these cells remains to be determined.

\section{Methods}

\section{PPIs}

The PPIs between SARS-CoV-2 and human proteins were collected from IMEX ${ }^{35}$, and those of SARS-CoV1 , from Biogrid ${ }^{36}$. On the other hand, the human interactome was generated from the compilation of information from several primary databases ${ }^{36-42}$. Only PPIs reported at least twice were considered.

\section{Proteomes and transcriptomes}

From the Normal tissue and RNA consensus tissue gene databases, deposited in the Protein Atlas (http://www.proteinatlas.org) ${ }^{43}$, proteomes corresponding to each cell of each tissue and transcriptomes of each tissue were generated. Only those tissue genes/proteins reported in both databases were considered (see Fig. 8). Proteins with an uncertain reliability score and genes with normalized expression (NX) values less than one were eliminated. For normal tissue data, values of 1, 2, 3 and 4 were assigned (provided there was a value $\geq 1$ in RNA consensus tissue gene data), for Not detected, Low, Medium, and High, respectively for expression value (level). For RNA consensus tissue gene data, the assigned NX 
values were used. The expression value and NX values of each cell were plotted by unsupervised hierarchical clustering in $\mathrm{R}^{44}$ using the gplots library ${ }^{45}$.

\section{Entrance tropism}

To identify which cells and tissues have the components to allow viral entry, the presence of ACE2, NRP1, BSG, TMPRSS2, CTSL, CTSB and FURIN proteins, or evidence of their gene expression, was assessed. Those with a combination of protein S-binding proteins (ACE2, NRP1 or BSG) and proteases necessary for protein S cleavage and membrane fusion (TMPRSS2, CTSL, CTSB or FURIN) were selected. Only those tissues with results present at both levels of information (proteome and transcriptome) were considered.

\section{Tropism at PPI level}

At this point, only the proteome (cell) data were processed. The interactomes (Virus-Human (V-H) and Human-Human $(\mathrm{H}-\mathrm{H})$ ) and proteomes of each cell were used. The interactomes of each cell of each tissue were generated considering the interactions of SARS-CoV-2 with human proteins $(\mathrm{V}-\mathrm{H})$, a second level of interaction $(\mathrm{H}-\mathrm{H})$, and the interactions between them. The networks were visualized with Cytoscape ${ }^{46}$, associating the Expression value and the level of interaction associated with the connectivity of each protein. Enrichment analysis was generated with Enrichr ${ }^{47}$ to identify the alterations of each cell, using the enrichR library of R to automate the procedures. Heat maps were performed with gplots.

Finally, centroids of each cell were identified by k-means, using the PPIs and the Proteome Expression value of each cell. Next, cells clusters susceptible to SARS-CoV-2 infection were identified according to its connectivity and autopsy reports.

\section{Declarations}

\section{Author contributions}

Conceptualization: DO-B; methodology: DO-B; software: DO-B; validation: DO-B and SZ; formal analysis: DO-B, SZ, MAMC, and RB; investigation: DO-B, SZ, MAMC, and RB; data curation: DO-B; writing-original draft preparation: DO-B, SZ, MAMC, and RB; writing-review and editing: DO-B, SZ, MAMC, and RB; visualization: DO-B; supervision: SZ and RB; project administration: RB. All authors reviewed the manuscript. All authors read and approved the final manuscript.

\section{Competing interests}

The authors declare no competing interests.

\section{Additional information}


Supplementary Information The online version contains supplementary material available at

Correspondence and requests for materials should be addressed to DO-B. or RB.

\section{References}

1. Gordon, D. E. et al. A SARS-CoV-2-Human Protein-Protein Interaction Map Reveals Drug Targets and Potential Drug-Repurposing. bioRxiv 2020.03.22.002386 (2020) doi:10.1101/2020.03.22.002386.

2. Li, J. et al. Virus-Host Interactome and Proteomic Survey Reveal Potential Virulence Factors Influencing SARS-CoV-2 Pathogenesis. Med (N Y) 2, 99-112.e7 (2021).

3. Stukalov, A. et al. Multilevel proteomics reveals host perturbations by SARS-CoV-2 and SARS-CoV. Nature 594, 246-252 (2021).

4. Khorsand, B., Savadi, A. \& Naghibzadeh, M. SARS-CoV-2-human protein-protein interaction network. Inform Med Unlocked 20, 100413 (2020).

5. Messina, F. et al. COVID-19: viral-host interactome analyzed by network based-approach model to study pathogenesis of SARS-CoV-2 infection. J Transl Med 18, 233 (2020).

6. Perrin-Cocon, L. et al. The current landscape of coronavirus-host protein-protein interactions. J Trans/ Med 18, 319 (2020).

7. Bojkova, D. et al. Proteomics of SARS-CoV-2-infected host cells reveals therapy targets. Nature 583, 469-472 (2020).

8. Kumar, N., Mishra, B., Mehmood, A., Athar, M. \& Mukhtar, M. S. Integrative Network Biology

Framework Elucidates Molecular Mechanisms of SARS-CoV-2 Pathogenesis. SSRN 3581857 (2020) doi:10.2139/ssrn.3581857.

9. Puelles, V. G. et al. Multiorgan and Renal Tropism of SARS-CoV-2. N Engl J Med 383, 590-592 (2020).

10. Cantuti-Castelvetri, L. et al. Neuropilin-1 facilitates SARS-CoV-2 cell entry and infectivity. Science 370, 856-860 (2020).

11. Singh, M., Bansal, V. \& Feschotte, C. A Single-Cell RNA Expression Map of Human Coronavirus Entry Factors. Cell Rep 32, 108175 (2020).

12. Bojkova, D. et al. SARS-CoV-2 and SARS-CoV differ in their cell tropism and drug sensitivity profiles. bioRxiv 2020.04.03.024257 (2020) doi:10.1101/2020.04.03.024257.

13. Sungnak, W. et al. SARS-CoV-2 entry factors are highly expressed in nasal epithelial cells together with innate immune genes. Nat Med 26, 681-687 (2020).

14. Yang, L. et al. A Human Pluripotent Stem Cell-based Platform to Study SARS-CoV-2 Tropism and Model Virus Infection in Human Cells and Organoids. Cell Stem Cell 27, 125-136.e7 (2020).

15. Li, M.-Y., Li, L., Zhang, Y. \& Wang, X.-S. Expression of the SARS-CoV-2 cell receptor gene ACE2 in a wide variety of human tissues. Infect Dis Poverty 9, 45 (2020). 
16. Xu, H. et al. High expression of ACE2 receptor of 2019-nCoV on the epithelial cells of oral mucosa. Int J Oral Sci 12, 8 (2020).

17. Zou, X. et al. Single-cell RNA-seq data analysis on the receptor ACE2 expression reveals the potential risk of different human organs vulnerable to 2019-nCoV infection. Front Med 14, 185-192 (2020).

18. Hikmet, F. et al. The protein expression profile of ACE2 in human tissues. Mol Syst Biol 16, e9610 (2020).

19. Bian, X.-W. \& COVID-19 Pathology Team. Autopsy of COVID-19 patients in China. Nat/ Sci Rev 7, 1414-1418 (2020).

20. Liu, J. et al. SARS-CoV-2 cell tropism and multiorgan infection. Cell Discov 7, 17 (2021).

21. von Stillfried, S. et al. SARS-CoV-2 RNA screening in routine pathology specimens. Microb Biotechnol (2021) doi:10.1111/1751-7915.13828.

22. Pontén, F. et al. A global view of protein expression in human cells, tissues, and organs. Mol Syst Biol 5, 337 (2009).

23. Gry, M., Oksvold, P., Pontén, F. \& Uhlén, M. Tissue-Specific Protein Expression in Human Cells, Tissues and Organs. (2010) doi:10.4172/JPB.1000153.

24. Huang, N. et al. Integrated Single-Cell Atlases Reveal an Oral SARS-CoV-2 Infection and Transmission Axis. medRxiv 2020.10.26.20219089 (2020) doi:10.1101/2020.10.26.20219089.

25. Zuo, Y. et al. Prothrombotic autoantibodies in serum from patients hospitalized with COVID-19. Sci Transl Med 12, eabd3876 (2020).

26. Wang, J. Y., Zhang, W., Roehrl, M. W., Roehrl, V. B. \& Roehrl, M. H. An autoantigen profile of human A549 lung cells reveals viral and host etiologic molecular attributes of autoimmunity in COVID-19. $J$ Autoimmun 120, 102644 (2021).

27. Wallukat, G. et al. Functional autoantibodies against G-protein coupled receptors in patients with persistent Long-COVID-19 symptoms. J Trans/ Autoimmun 4, 100100 (2021).

28. Boscolo-Rizzo, P. et al. Sequelae in adults at 12 months after mild-to-moderate coronavirus disease 2019 (COVID-19). Int Forum Allergy Rhinol (2021) doi:10.1002/alr.22832.

29. Pelletier, J., Thomas, G. \& Volarević, S. Ribosome biogenesis in cancer: new players and therapeutic avenues. Nat Rev Cancer 18, 51-63 (2018).

30. Chen, J.-Y. et al. Interaction between SARS-CoV helicase and a multifunctional cellular protein (Ddx5) revealed by yeast and mammalian cell two-hybrid systems. Arch Virol 154, 507-512 (2009).

31. Wang, J., Pendurthi, U. R., Yi, G. \& Rao, L. V. M. SARS-CoV-2 infection induces the activation of tissue factor-mediated coagulation by activation of acid sphingomyelinase. Blood blood.2021010685 (2021) doi:10.1182/blood.2021010685.

32. Vinayagam, S. \& Sattu, K. SARS-CoV-2 and coagulation disorders in different organs. Life Sci 260, 118431 (2020).

33. Peiris, J. S. M. \& Cheung, C. Y. The macrophage in the pathogenesis of severe acute respiratory syndrome coronavirus infection. Hong Kong Med J 15 Suppl 6, 21-25 (2009). 
34. Yilla, M. et al. SARS-coronavirus replication in human peripheral monocytes/macrophages. Virus Research 107, 93-101 (2005).

35. Perfetto, L. et al. The IMEx coronavirus interactome: an evolving map of Coronaviridae-host molecular interactions. Database (Oxford) 2020, baaa096 (2020).

36. Oughtred, R. et al. The BioGRID interaction database: 2019 update. Nucleic Acids Res 47, D529D541 (2019).

37. Sprinzak, E., Cokus, S. J., Yeates, T. O., Eisenberg, D. \& Pellegrini, M. Detecting coordinated regulation of multi-protein complexes using logic analysis of gene expression. BMC Syst Bio/ 3, 115 (2009).

38. Orchard, S. et al. The MIntAct project-IntAct as a common curation platform for 11 molecular interaction databases. Nucleic Acids Res 42, D358-363 (2014).

39. Keshava Prasad, T. S. et al. Human Protein Reference Database-2009 update. Nucleic Acids Res 37, D767-772 (2009).

40. Clerc, O. et al. MatrixDB: integration of new data with a focus on glycosaminoglycan interactions. Nucleic Acids Res 47, D376-D381 (2019).

41. Licata, L. et al. MINT, the molecular interaction database: 2012 update. Nucleic Acids Res 40, D857861 (2012).

42. Jassal, B. et al. The reactome pathway knowledgebase. Nucleic Acids Res 48, D498-D503 (2020).

43. Thul, P. J. et al. A subcellular map of the human proteome. Science 356, eaal3321 (2017).

44. R Core Team. R: A language and environment for statistical computing. R Foundation for Statistical Computing Vienna, Austria https://www.r-project.org/.

45. Warnes GR et al. gplots: Various R Programming Tools for Plotting Data version 3.1.1 from CRAN. https://rdrr.io/cran/gplots/ (2012).

46. Shannon, P. et al. Cytoscape: a software environment for integrated models of biomolecular interaction networks. Genome Res 13, 2498-2504 (2003).

47. Kuleshov, M. V. et al. Enrichr: a comprehensive gene set enrichment analysis web server 2016 update. Nucleic Acids Res 44, W90-97 (2016).

\section{Figures}




\section{Normal RNA consensus tissue data tissue gene data}

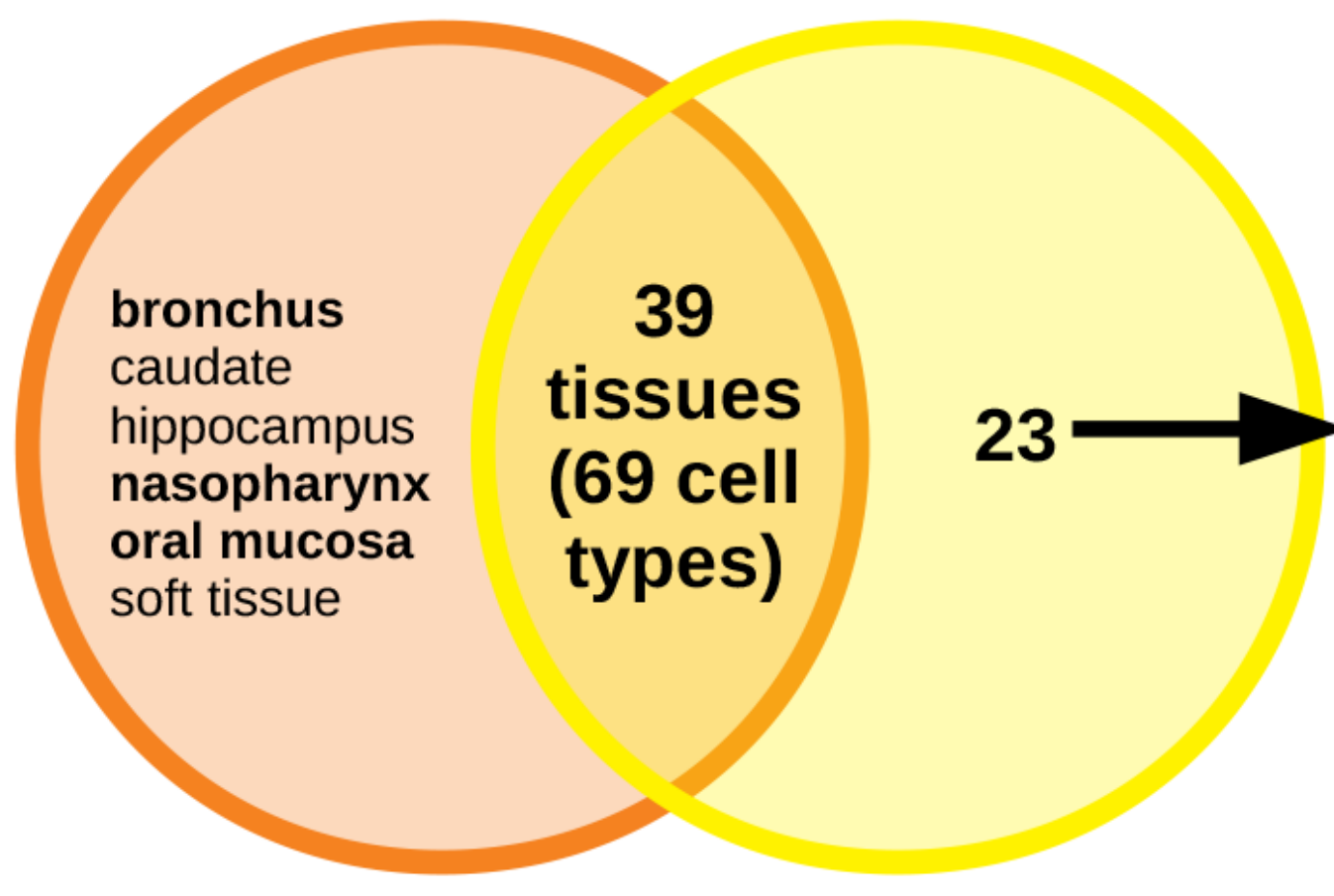

amygdala

B-cells

basal ganglia corpus callosum dendritic cells ductus deferens granulocytes hippocampal formation hypothalamus midbrain monocytes NK-cells olfactory region pituitary gland pons and medulla retina spinal cord substantia nigra

T-cells thalamus thymus tongue total PBMC

\section{Figure 1}

Initial results. Tissues that shared a set of the seven cell entry proteins/genes. It is observed that in the databases used, for six cell types there are no reports at the tissue level (transcriptome) and for 23 tissues no cells are reported (proteome). 
a
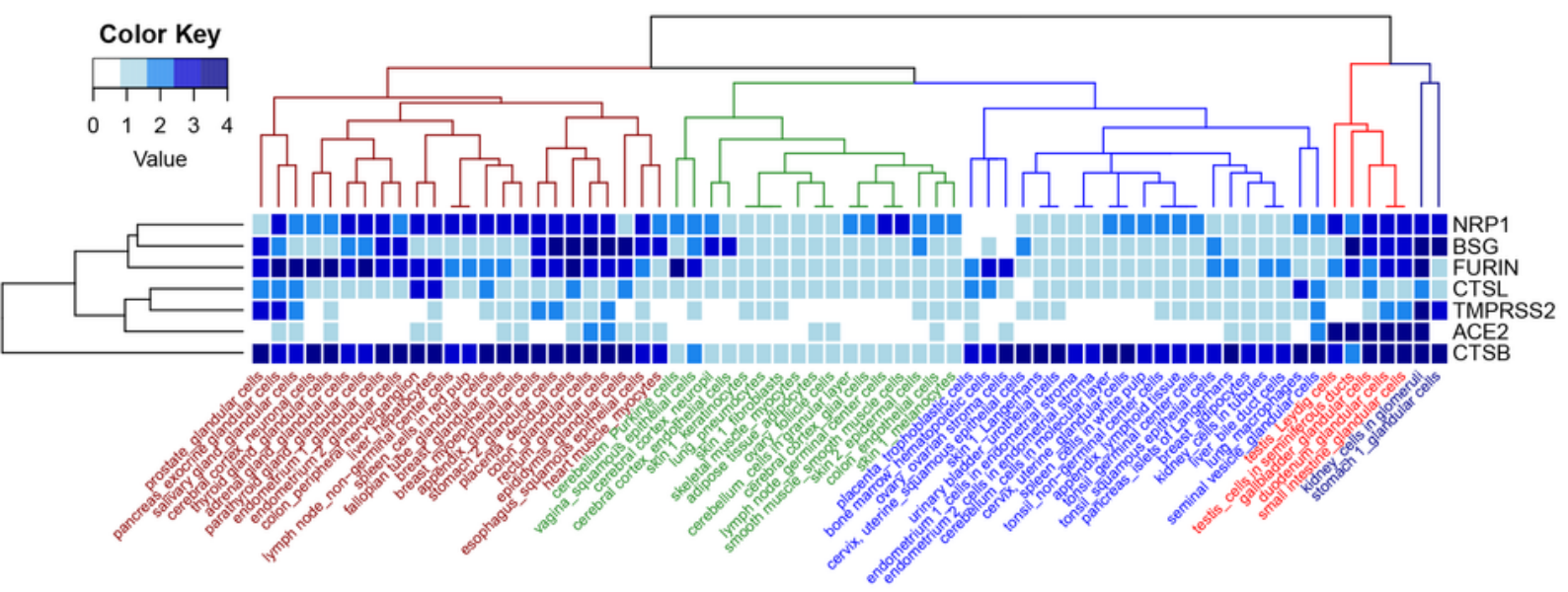

b

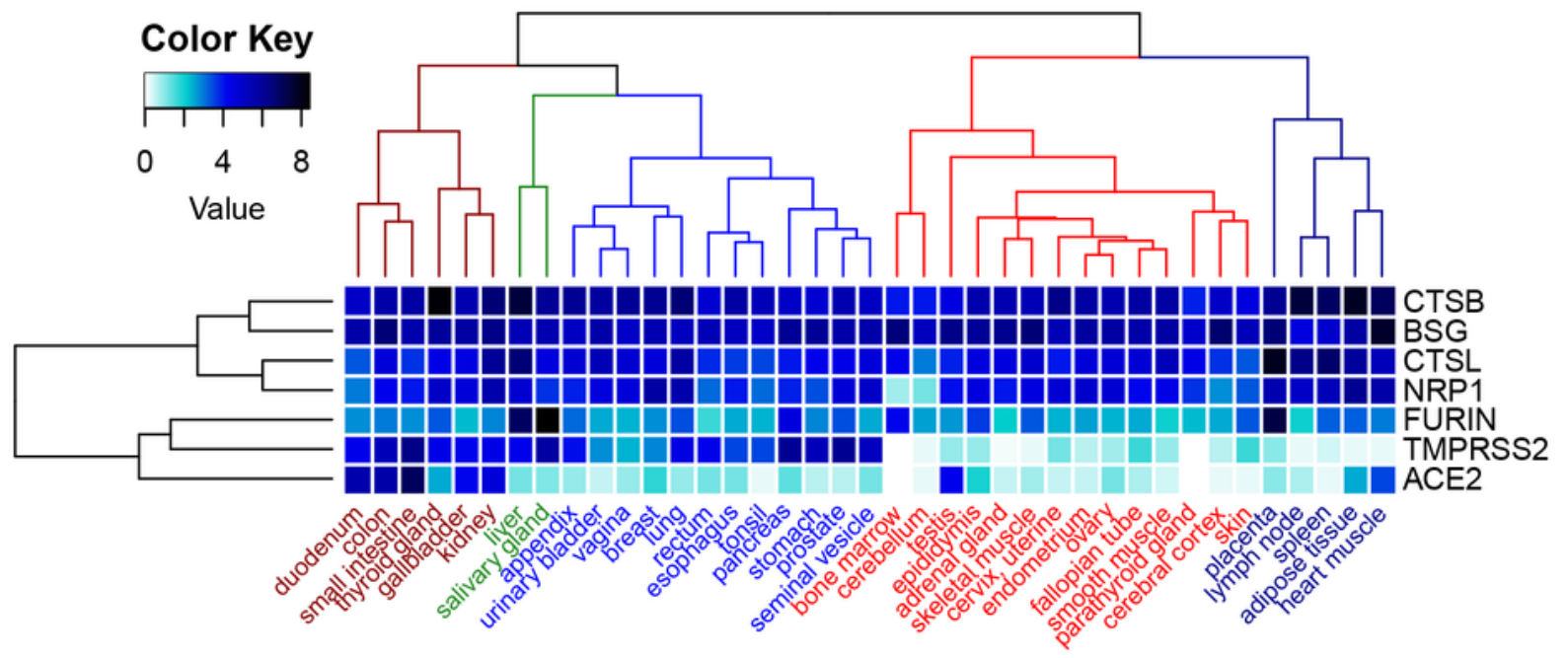

C

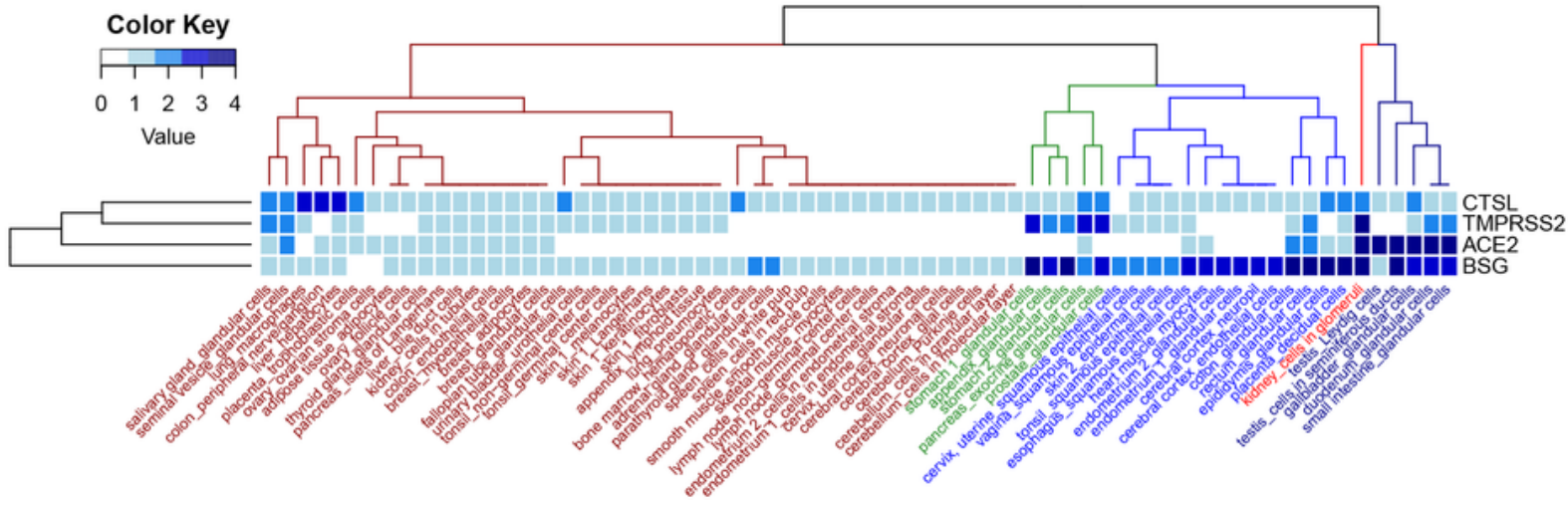

Figure 2

Cell and tissue heatmap based on potential input genes/proteins. (a) SARS-CoV-2, 69 cell types and seven proteins. In the red and dark blue branches and later in the dark red branch, the cell types with the highest protein presence are concentrated. Green and blue branches group those cell types with lower presence, as well as those without ACE2 or TMPRSS2 (also in the dark red branch), mainly. (b) SARS-CoV2, 39 tissues and seven messengers. Consistent with what is reported in the figure above, ACE2 and 
TMPRSS2 have the lowest expression, followed by FURIN. Of the 39 tissues, bone marrow, cerebellum and parathyroid gland have the lowest expression of ACE2 and TMPRSS2. (c) SARS-CoV-1, 69 cell types and 4 proteins. The dark red branch is where the cells with the lowest presence of protein or mRNA are grouped; in the central part, the 14 removed cells are observed.
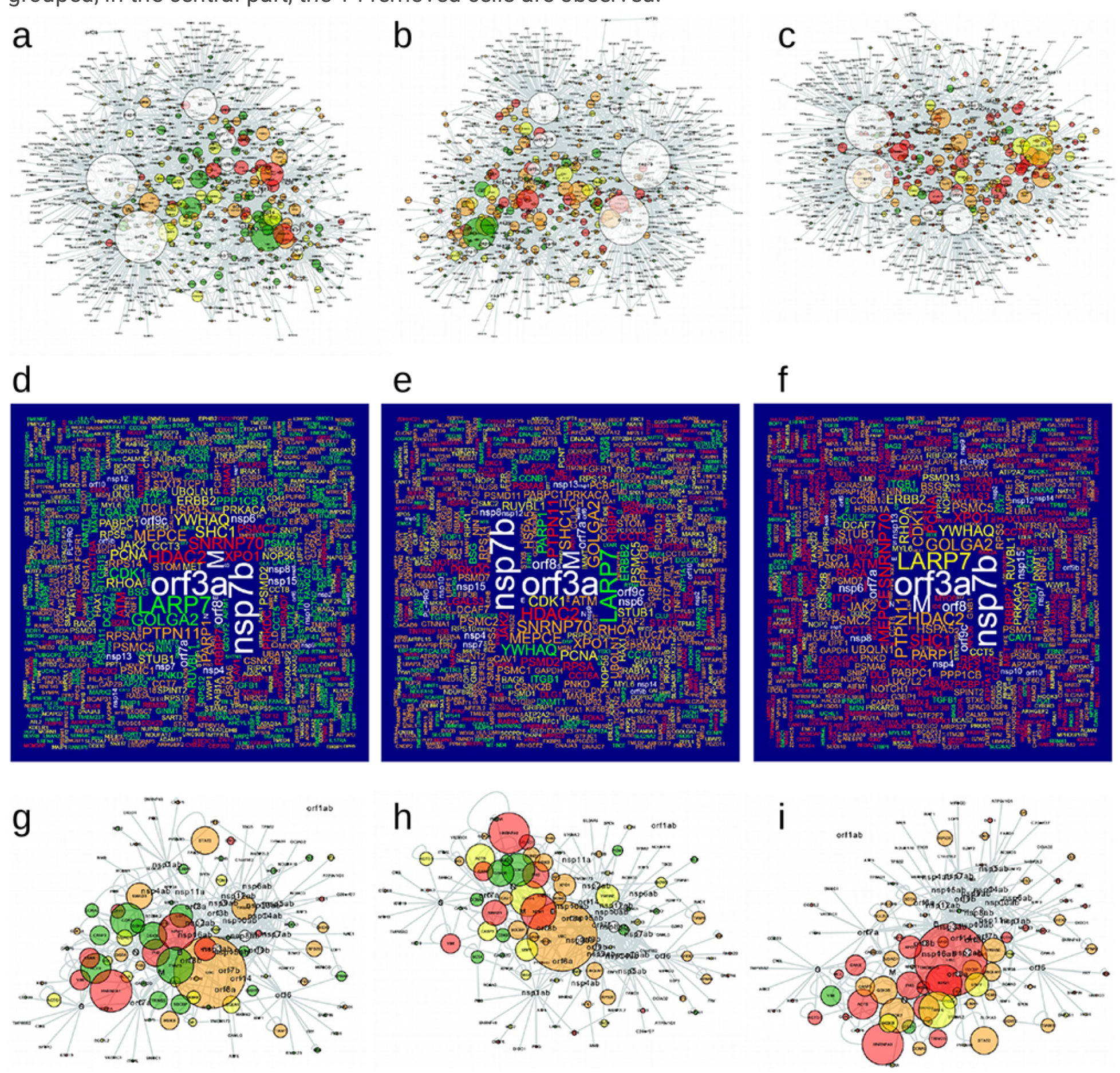

Figure 3

$\mathrm{V}-\mathrm{H}$ networks and their respective wordclouds. (a) and (d) lung (pneumocytes); (b) and (e) lung (macrophages); (c) and (f) small intestine (glandular cells), in SARS-CoV-2; (g), (h) and (i) networks in SARS-CoV-1, same order as the previous ones. The size of the circles indicates the connectivity and the color to the Expression value, 1: green, 2: yellow, 3: orange, 4: red and in white the viral proteins. 

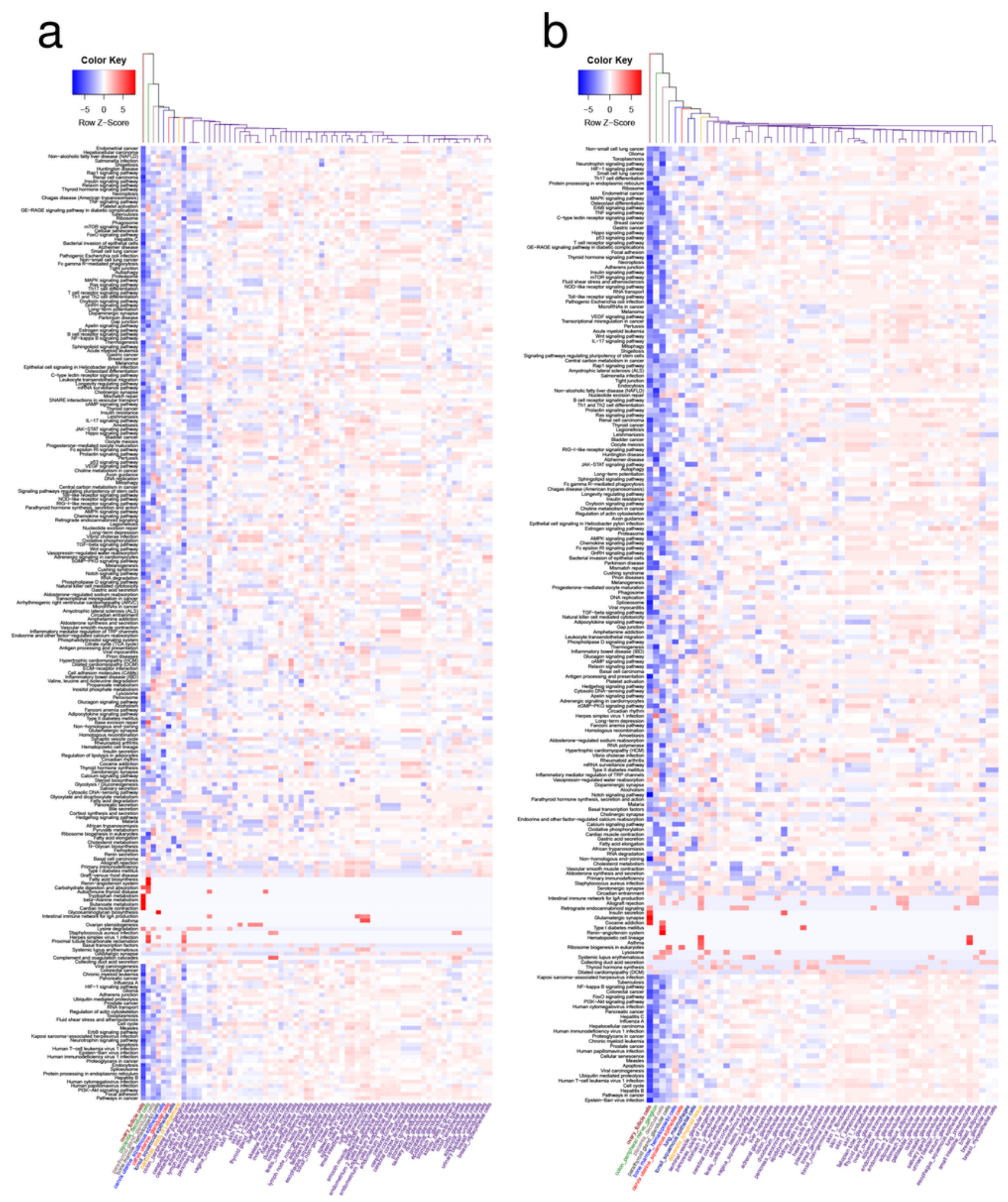

\section{Figure 4}

Enrichment of results, KEGG heatmap. (a) Signaling pathways in SARS-CoV-2; (b) signaling pathways in SARS-CoV-1. The blue color identifies a higher $p$-value and the red color a lower value (using a logarithmic transformation). A gradient of involvement of the signaling pathways, and therefore of the cells, is identified in both heatmaps from left to right (less altered to more altered, respectively). 

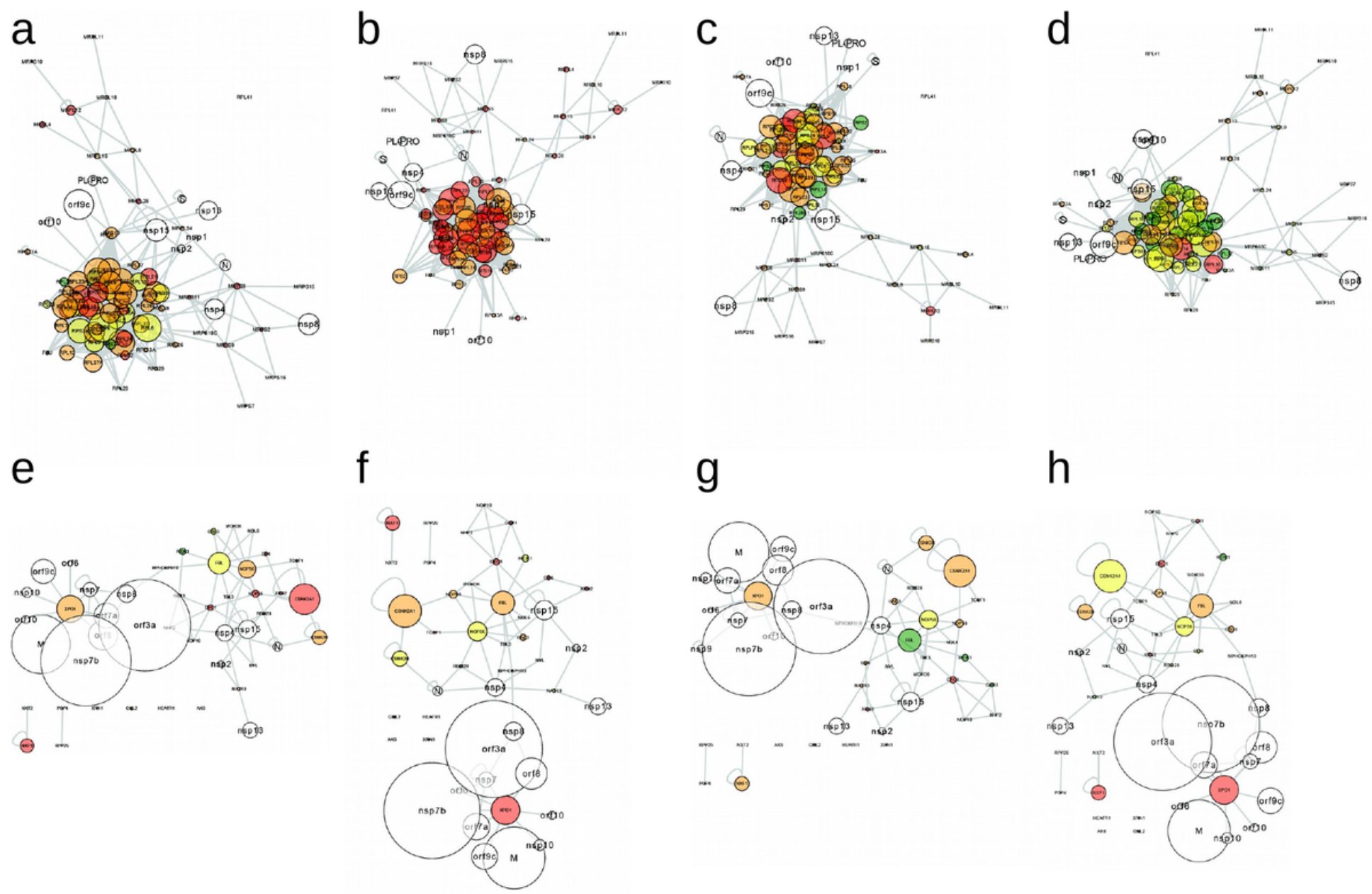

Figure 5

Specific networks. (a), (b), (c) and (d) ribosome pathway networks; (e), (f), (g) and (h) networks of the ribosome synthesis pathway. Kidney (cells in tubules), small intestine (glandular cells) and lung (macrophages and pneumocytes), respectively. The color code and size nodes are the same as in Fig. 4. In the ribosome pathway there are 11 viral proteins ( $N$, nsp4, PL-PRO, nsp2, nsp8, nsp13, orf9c, S, nsp1, orf10, and nsp15) that interact with proteins of this pathway. In Ribosome biogenesis in eukaryotes pathway there are 16 viral proteins (nsp7b, N, orf7a, nsp4, nsp2, nsp8, nsp13, nsp7, orf8, nsp10, orf9c, M, orf6, orf10, nsp15, and orf3a) that interact with proteins in this pathway; among them the 3 proteins with a high connectivity of SARS-CoV-2: $M, n s p 7 b$, and orf3a. These data are for the 4 cells shown. 
a

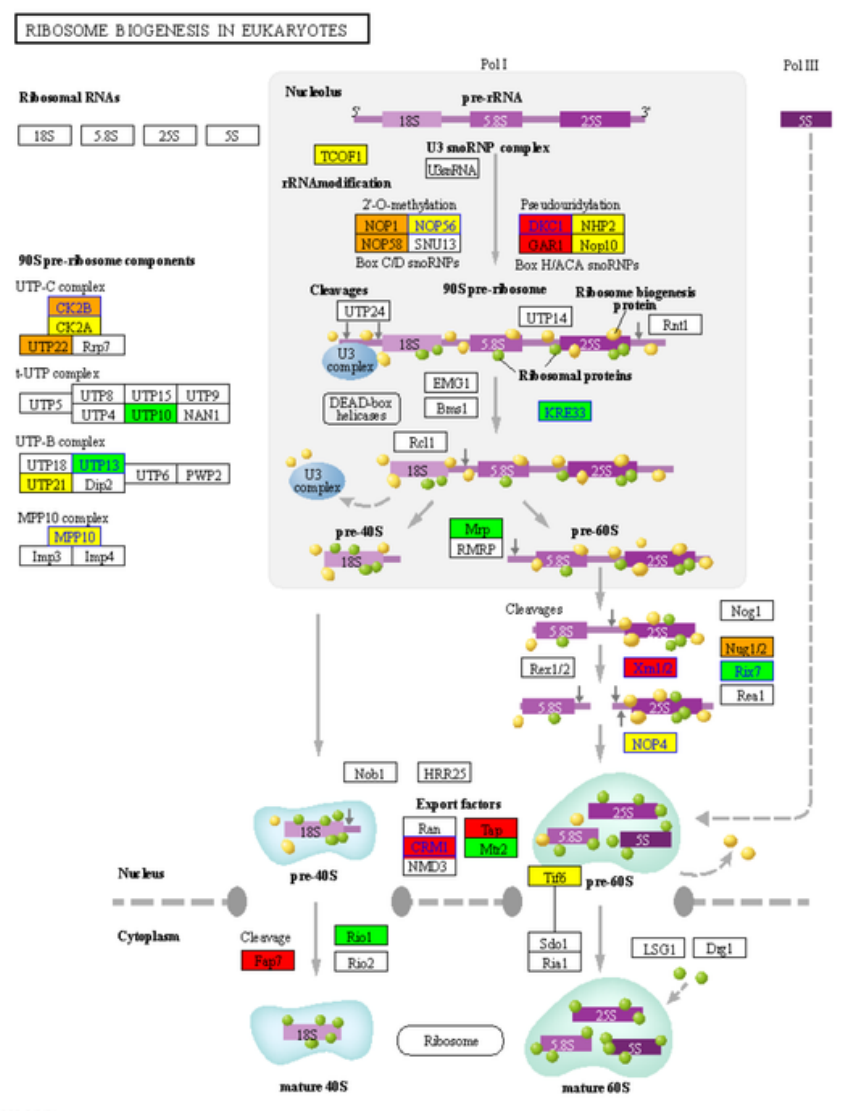

b

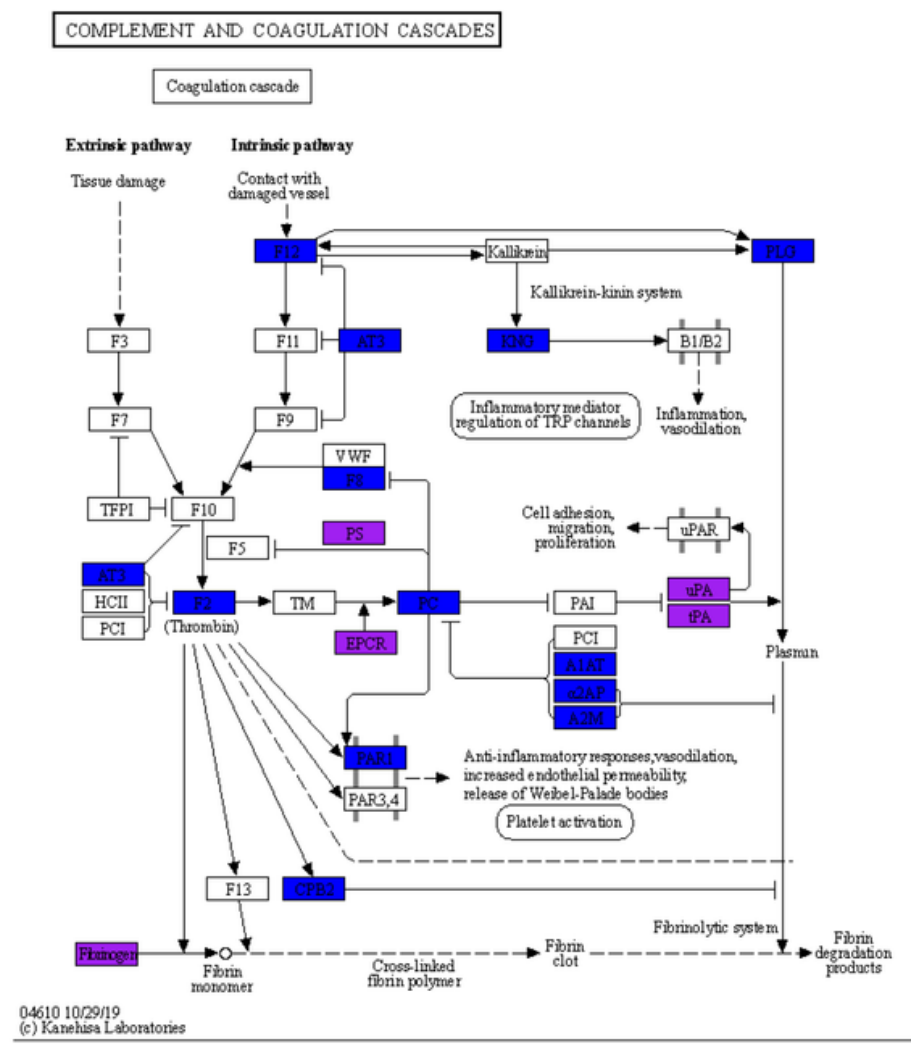

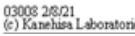

\section{Figure 6}

Signaling pathways KEGG. (a) Signaling pathway for the synthesis of ribosomes in lung (pneumocytes), 10 proteins with direct interaction with name and line blue. 30 of 101 proteins are reported. he colors code associated to expression value was used. (b) Coagulation cascade and the 19 proteins (39 including those of the complement system) that can interact directly (purple), 6 proteins, or indirectly (blue), 13 proteins, with SARS-CoV-2 proteins in blood. Only the coagulation cascade is shown. 
a

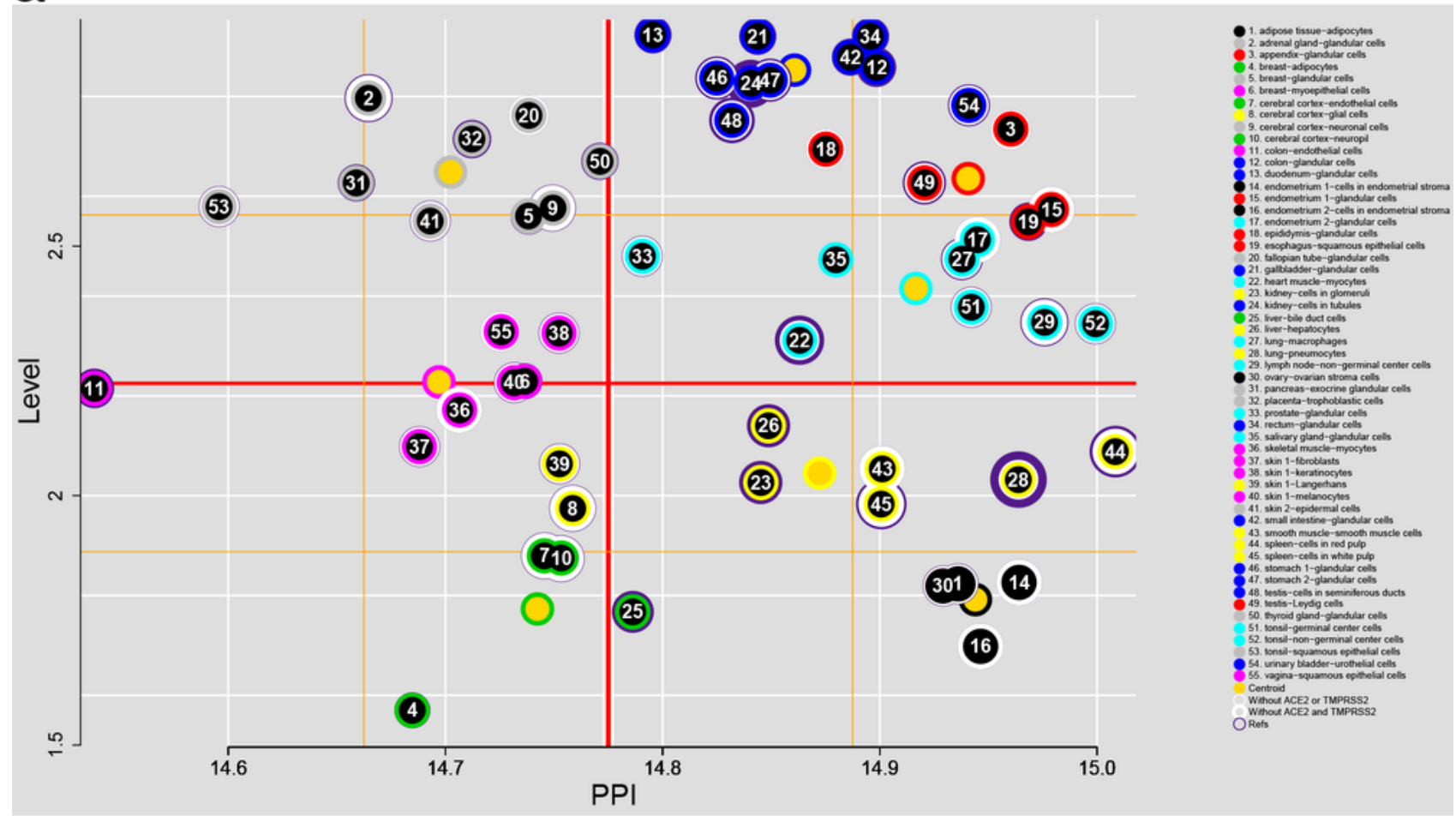

b

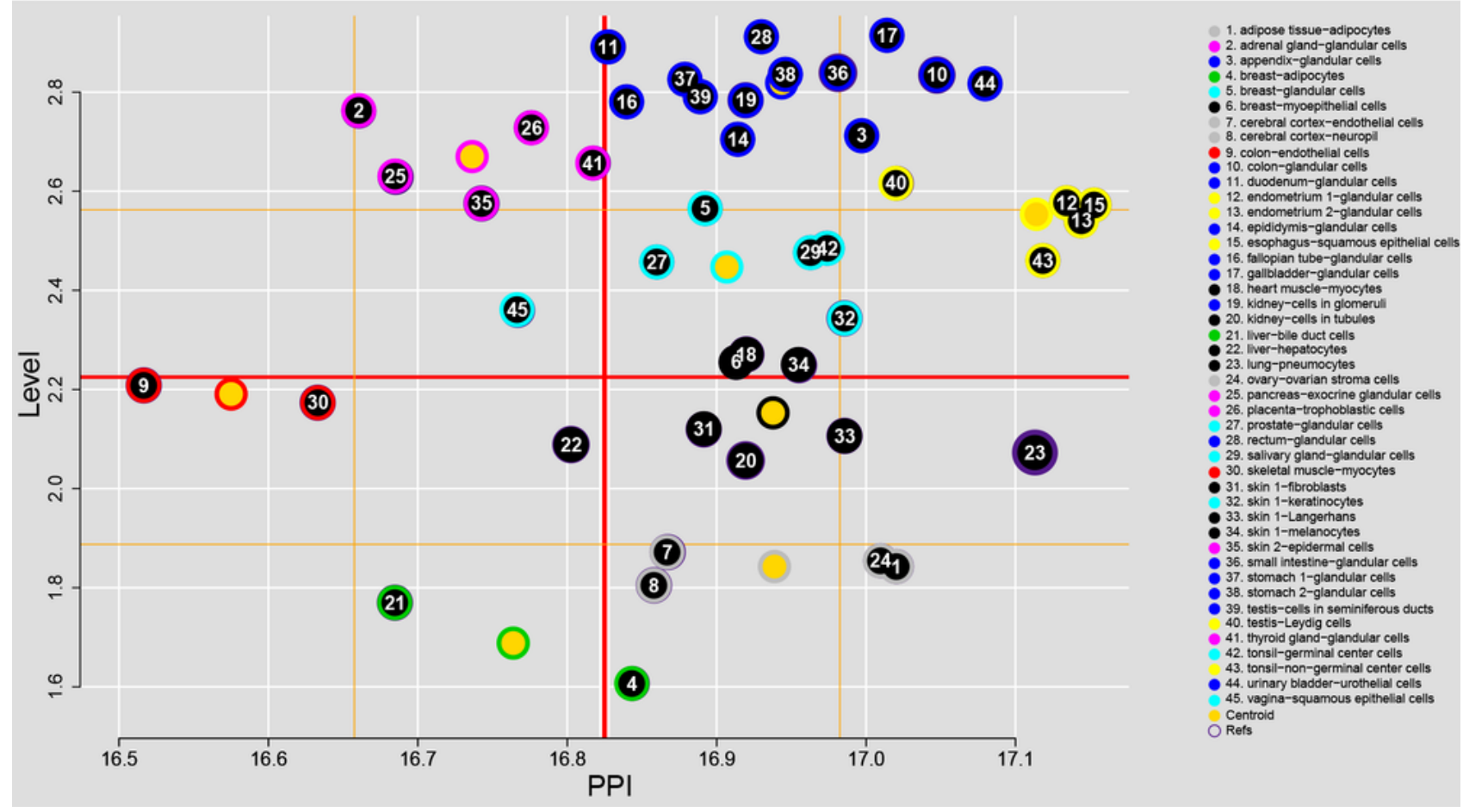

Figure 7

K-Means of the cells and their potential of infection. (a) SARS-CoV-2; at the top of quadrant 4, the cells most reported in the literature as infected in autopsies are identified (width purple ring), followed by quadrant 1. (b) SARS-CoV-1; pneumocytes are found in the same region as in A (upper side of quadrant 4, black group). Quadrant 1: high expression value (Level) and high PPI (upper right side); quadrant 2: high expression value and low PPI (upper left side); quadrant 3: low expression value and low PPI (bottom 
left); and quadrant 4: low expression value and high PPI (lower right side). As expected, glandular cells are mainly concentrated in the upper quadrants, gray, blue, red, and cyan cluster in (a) and lilac, blue, cyan, and yellow in (b).

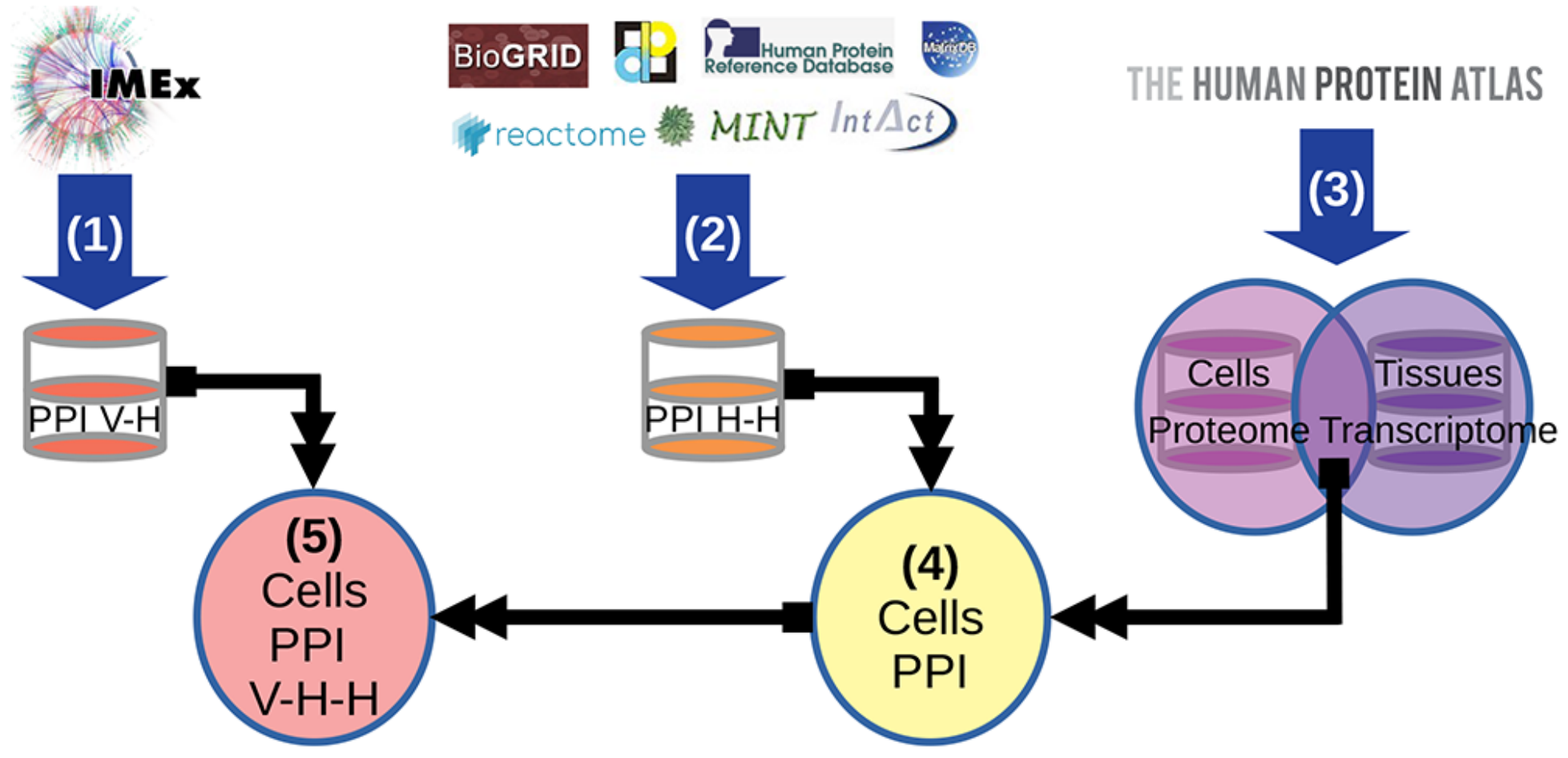

Figure 8

Research strategy. 1: Obtaining the PPI of the SARS-CoV-2 - Human (V-H) from IMEx; 2: Integration of human-specific PPIs from 7 primary databases $(\mathrm{H}-\mathrm{H})$; 3: Obtaining proteomes and transcriptomes, selecting those results that are reported at both levels; 4: Generation of human PPI $(\mathrm{H}-\mathrm{H})$ for each cell; 5 : Obtaining the PPI of the viral infection $(\mathrm{V}-\mathrm{H}-\mathrm{H})$ of each cell. Images were taken from https://www.imexconsortium.org/, https://dip.doe-mbi.ucla.edu/dip/Main.cgi, https://www.ebi.ac.uk/intact/, https://mint.bio.uniroma2.it/, https://thebiogrid.org/, https://www.hprd.org/, http://matrixdb.univ-lyon1.fr/, https://reactome.org/ and http://www.proteinatlas.org.

\section{Supplementary Files}

This is a list of supplementary files associated with this preprint. Click to download.

- Supplementarylnformation.pdf

- SupplementaryTables.xlsx 